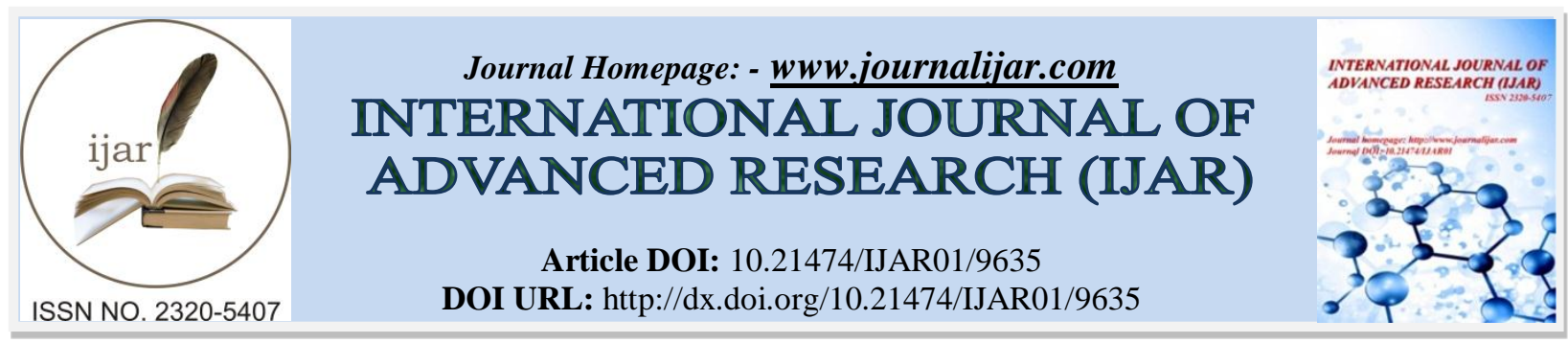

RESEARCH ARTICLE

\title{
UKOPAJI WA LUGHA: ETIMOLOJIA NA MABADILIKO YA MAANA NA UTAMKAJI WA MSAMIATI WA KIARABU KATIKA KISWAHILI.
}

Dkt. Hamisi Babusa.

Chuo Kikuu cha Kenyatta

\section{Manuscript Info}

Manuscript History

Received: 02 July 2019

Final Accepted: 04 August 2019

Published: September 2019

\begin{abstract}
Pindi wazungumzaji wa lugha mbili wanapotagusana na kuingiliana basi moja kwa moja lugha hizo huweza kukopana na kuathiriana kifonolojia, kimsamiati na kisarufi. Waarabu walipofika pwani ya Afrika Mashariki, walikumbana na Waswahili na hapo ndipo Kiswahili kikakopa maneno na sauti za kiarabu ambazo hazikuwepo katika lugha ya Kiswahili. Pili, Kiswahili pia kikachukua baadhi ya sauti za kiarabu na kuzibadili jinsi zinavyotamkwa. Makala haya yanatafiti na kuainisha etimolojia ya baadhi ya maneno ya kiarabu yanayopatikana katika lugha ya Kiswahili na jinsi sauti za kiarabu zilivyokopwa na kubadilishwa na kuswahilishwa.na pili namna baadhi ya maneno yalivyobadilishwa maana asilia baada ya kukopwa kutoka lugha ya Kiarabu. Nadharia za mfumo na semantiki tambuzi za msamiati zilitumika.
\end{abstract}

Copy Right, IJAR, 2019,. All rights reserved.

\section{Introduction:-}

Kiswahili kilipotagusana na lugha ya kiarabu, kiliazima vitu viwili. Kiliazima maneno mengi ya kiarabu na kuyaswalihisha na pili ikaazima sauti za kiarabu ambazo zilikuja na baadhi ya maneno hayo. Uingiaji wa maneno na sauti za kiarabu katika lugha ya Kiswahili kulisababisha mabadiliko ya lugha ya aina mbili. Aina ya kwanza ya mabadiliko ni ubadilishaji au urahisishaji wa sauti za kiarabu ili ziweze kutamkwa na wazungumzaji wa Kiswahili ambayo ni lugha ya Kibantu. Pili ubadilikaji wa maana asilia ya baadhi ya maneno ya kiarabu yalipoingizwa katika lugha ya Kiswahili. Makala haya yatajadili ukopaji wa lugha kutoka kiarabu hadi ndani ya Kiswahili na jinsi ya ubadilikaji wa sauti ulivyofanyika na pia ubadilikaji wa maana asilia wa baadhi ya maneno ya kiarabu yaliyokopwa na lugha ya Kiswahili.

Makala haya yametumia nadharia mbili. Ya kwanza ni nadharia ya mfumo inayosimamia mabadiliko ya sauti za maneno yaliyokopwa kutoka kwa lugha ya Kiarabu. Kulingana na Mulder na Hervy (1975) nadharia hii inachukulia lugha kama mfumo mkubwa ambao una mifumo mingi ndani. Wanasema kuwa lugha ni mfumo wenye mifumo mitano ambayo ni semantiki, pragmatiki, sintaksia. Mofolojia na fonolojia. Makala haya yatajikita katika fonolojia kutazama namna sauti zinavyobadilika.

Nadharia ya pili ni ya Semantiki tambuzi za msamiati (cognitive lexical semantics) Kulingana na Paradis (2012), nadharia hii inajishughulisha mabadiliko ya maana katika maneno ambayo ni mojawapo ya mada ya makala haya.

Corresponding Author:-Dkt. Hamisi Babusa.

Address:-Chuo Kikuu cha Kenyatta. 


\section{Mabadiliko ya sauti katika maneno ya kiarabu yaliyokopwa.}

Maneno mengi ya kiarabu ambayo yalikopwa na lugha ya Kiswahili, mbali na kupitia mabadiliko ya kuyavokalisha na kufuata mfumo wa maneno ya Kiswahili, pia yalipitia mabadiliko ya sauti zao asili zilizobadilishwa. Sauti hizo zilibadilishwa kwa sababu hazikuwapo kabisa katika lugha ya Kiswahili na pia hata kama zingekopwa, zingeleta matatizo ya utamkaji kwa wazungumzaji wa Kiswahili ambao hawakuzoea kutamka sauti hizo za kiarabu. Mifano ifuatayo inadhihirisha mabadiliko ya utamkaji wa baadhi ya maneno ya Kiarabu yaliyoingizwa katika lugha ya Kiswahili.

Upishanishaji Wa ي (Yee) Ya Kiarabu Na Vokali Ya Kiswahili.

ي (Yee) au /y/ ni sauti au konsonanti ya mwisho katika alfabeti ya Kiarabu. Kuna baadhi ya maneno ya Kiarabu ambayo huwa na (yee) iliyotiwa shadda. Kwa mfano
MANIYYI منيّ
نبيّ NABIYYI
HAYYU حئ طئ
طيب TWAYYB
MAYYITU ميّت طيّ

Maneno hayo yalipokopwa na Kiswahili ي (yee) ilidondoshwa na badala yake kukawekwa vokali/i/.

MANIYYI likawa MANII

NABIYYI likawa NABII.

HAYYU likawa HAI

TWAYYIB likawa taib

MAYYITU likawa MAITI nk

\section{Upishanishaji Wa $\varepsilon($ ('Ain) Ya Kiarabu Na Vokali Ya Kiswahili}

$\varepsilon$ ('ain) ni konsonanti ya kumi na nane katika alfabeti ya kiarabu. Baadhi ya maneno ya kiarabu yaliyo na hii saurti ni kama:

'Aib عيب

Mu’allim معلم

'Ahdi عهt

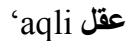

tis'a تسعة

Maneno hayo yalipokopwa na kuingizwa kwenye Kiswahili, $\varepsilon$ 'ain ya kiarabu ambayo kwa waafrika itakuwa ngumu kuitamka, ilidondoshwa na badala yake kukawekwa vokali.

'aib likawa AIBU

mu'allim likawa MWALIMU

'ahdi likawa AHADI

'aqli likawa AKILI

tis'a likawa TISA

\section{Ubadilikaji Wa b (Twee) Ya Kiarabu Na Kuwa /T/ Kiswahili}

ubadilikaji mwengine wa sauti ni ule ambapo neno la kiarabu lenye konsonanti ya Kiarabu b (twee) hubadilika na kuwa /t/ baada ya kukopwa hadi lugha ya Kiswahili. kwa mfano, maneno yafuatayo ni ya kiarabu:

طWAYYIB طيب TWA

طWAARIQ طآب TW

maneno hayo yalipokopwa na kuingizwa kwenye lugha ya Kiswahili, konsonanti b (twee) ilidondoshwa na kubadilishwa na /t/ na maneno hayo yakawa TARIKI na TAIB katika Kiswahili.

Ubadilikaji Wa ص (Swaad) Ya Kiarabu Na Kuwa /S/ Katika Kiswahili.

Maneno ya Kiarabu ambayo yana konsonanti ص (swaad) yakiingia kwenye lugha ya Kiswahili, ص (swaad) hubadilika na kuwa /s/. Kwa mfano:

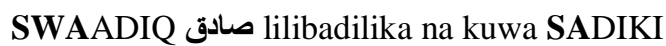

SWAGHYR صغير lilibadilika na kuwa SAGHIRI

SWIRAAT صرآت lilibadilika na kuwa SIRATI 


\section{Ubadilikaji Wa قa (Qaaf) Ya Kiarabu Na Kuwa /K/ Katika Kiswahili}

Katika alfabeti za Kiarabu, kuna (kaaf)/k/ na kuna ق (qaaf). ق (qaaf) ni konsonanti ya ishirini na moja katika alfabeti ya Kiarabu. ni sauti ambayo hutamkwa nyuma ya /k/ yaani kwa mguso wa kaakaa laini na sehemu ya nyuma ya ulimi. Wazungumzaji wa Kibantu hawakuwa na hii sauti na maneno yenye sauti hii ya Kiarabu yalipokopwa na kutiwa Kiswahilini, ق (qaaf) ilibadilika na kuwa /k/. kwa mfano:

SWAADIQ صادق lilibadilika na kuwa SADIKI

TWAARIQ طآرق lilibadilika na kuwa TARIKI

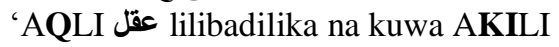

WARAQA ورقه lilibadilika na kuwa WARAKA

WAQTI وقت lilibadilika na kuwa WAKATI

\section{Ubadilikaji Wa (Dhwee) Ya Kiarabu Na Kuwa /Dh/ Katika Kiswahili}

ظ (dhwee) nayo ni herufi ya kumi na saba katika alfabeti ya Kiarabu. Maneno yenye hii sauti yalipokopwa na kuingiswa kwenye Kiswahili ל (dhwee) ilibadilishwa na badala yake ikawa /dh/. Kwa mfano:

DHWAALIM ظآم lilibadilika na kuwa DHALIMU

NADHWIIF نظيف lilibadilika na kuwa NADHIFU

\section{Udondoshaji Wa Vokali Ya Kiarabu Katika Kiswahili}

baadhi ya maneno ya Kiarabu yana sauti iliyovutwa ama kuwekwa irabu mbili. Yanapokopwa na kuingizwa Kiswahilini, vokali moja hudondoshwa au hukatwa na kubakisha moja. Kwa mfano:

TWAARIQ طآرق lilibadilika na kuwa TARIKI

DHWAALIM ظآلم طارث lilibadilika na kuwa DHALIMU

NADHWIIF نظيف lilibadilika na kuwa NADHIFU

\section{Ubadilikaji Wa ض ('Llwad) Ya Kiarabu Na Kuwa /Dh/ Katika Kiswahili}

ض ('llwad) ni konsonanti ya kumi na tano katika alfabeti ya Kiarabu. Wataalam wengi huiweka hii sauti kama /d/ lakini si /d/. Ni sauti inayotamkwa kwa sehemu ya mbele ya ulimi (si ncha ya ulimi) na meno. Sauti hii huleta mushkili kuitamka hasa kwa mzungumzaji ambaye hajui lugha ya Kiarabu. Waswahili hawana hii sauti kwahivyo wakatafuta sauti inayokaribiana na wakapata/dh/ na mara moja maneno yenye ض ('llwad) ya Kiarabu yakabadilika na kutamkwa na /dh/ baada ya kuingizwa kwenye lugha ya Kiswahili. Mfano ni:

AR'LLI أرض hubadilika na kuwa ARDHI

\section{'LLARhubadilika na kuwa برض AB DHORUBA}

\section{Ubadilikaji Katika /H/ Inayoghuna Na /H/ Isiyoghuna}

Katika lugha ya Kiarabu, kuna /h/ aina mbili. Ya kwanza ni $\tau$ /h/ isiyoghuna na hutamkwa sehemu ya juu ya koo. Kisha kuna $\$ / \mathrm{h} /$ inayoghuna ambayo hutamkwa kifuani. Katika lugha ya Kiswahili, kuna /h/ moja tu, ile ambayo hutamkwa kooni. Yale maneno ya Kiarabu yenye sauti $\$ / \mathrm{h} /$ inayoghuna yakikopwa na kuingizwa kwenye lugha ya Kiswahili, sauti hii hubadilishwa na kuwa /h/ isiyoghuna. kwa mfano:

'AHDI عه lilibadilika na kuwa AHADI

JUHDI جها lilibadilika na kuwa JUHUDI

\section{Udondoshaji Wa Sauti Za Mwisho Za Maneno Ya Kiarabu}

Baadhi ya maneno ya Kiarabu yana sauti za mwisho ambazo aghalabu hutumiwa kama kiambishi cha mwisho cha neno na aghalabu huwa ni $\bullet$ (hamza) na $\ddot{\partial} / \mathrm{t} /$. kwa mfano:

HAYAAI حياء hamza hudondoshwa na vokali moja baada ya /y/ ikaondolewa na neno likabadilika na kuwa HAYA SWADAQAATU صدقات /t/ ya mwisho hudondoshwa na vokali moja baada ya /q/ ikaondolewa na /qaaf/ ikawa /k/ na neno likabadilika na kuwa SADAKA

RISAALATU رسالة/t/ ya mwisho hudondoshwa na vokali moja baada ya /s/ ikaondolewa na neno likabadilika na kuwa RISALA WARAQATU ورقة/t/ ya mwisho hudondoshwa na /qaaf/ ikawa/k/ na neno likabadilika na kuwa WARAKA

\section{Uhamishaji Wa Muundo Wa Maneno Ya Kiarabu Hadi Kiswahili}

Maneno ya Kiswahili aghalabu huwa yanapangwa KVKV. kwa mfano KAKA, DADA, MAMA NK. Maneno mengi ya Kiarabu yalipokopwa aghalabu huwa hayafuati mfumo huu wa lugha ya Kiswahili. Huwa yana muundo wa KVKKV nk. Lakini yanapokopwa na kutiwa kwenye Kiswahili, huwa yanavokalishwa na kuwekwa katika muundo wa KVKV. Kwa mfano: 
'AHDI (KKKV) عهد lilibadilika na kuwa AHADI (VKVKV)

JUHDI (KVKKV) جهد lilibadilika na kuwa JUHUDI(KVKVKV)

Lakini kuna baadhi ya maneno yenye muundo wa lugha ya Kiarabu ambayo yalipokopwa katika Kiswahili, ilibidi muundo ubaki vilevile na kufanya lugha ya Kiswahili kuwa na muundo mpya wa maneno kutoka kwenye lugha ya Kiarabu. Kwa mfano:

AR'LLI أرض (VKKV) hubadilika na kuwa ARDHI (VKKV)

KHAMSIIN خمسين (KVKKVVK) Hili neno lilipoingizwa katika Kiswahili lilibaki na muundo wake wa Kiarabu na mabadiliko yake yalikuwa akali, yaani /kh/ ilibadilishwa na kuwa /h/, vokali moja baada ya /s/ ilidondoshwa na pia vokali ikaongezwa mwisho wa neno. Lakini hakukuwekwa vokali baada ya $/ \mathrm{m} /$ na muundo wa Kiarabu ukaingizwa katika lugha ya Kiswahili na neno likawa HAMSINI (KVKKVKV)

\section{Mabadiliko ya maana ya baadhi ya maneno ya kiarabu yaliyokopwa.}

Maneno mengi ya Kiarabu yaliyokopwa na kutiwa Kiswahilini aghalabu yalibaki na maana yake asilia kutoka Kiarabu. Lakini baadhi ya maneno yalibadilishwa maana baada ya kukopwa na kuchukua maana nyengine katika lugha ya Kiswahili. Sehemu hii itatalii baadhi ya maneno yaliyobadilishwa maana baada ya mkopo.

\section{Dari}

ni neno la Kiswahili lililokopwa kutoka kwenye lugha ya Kiarabu دار. Maana asilia ya hili neno kwa Kiarabu ni NYUMBA. mfano likitumiwa katika neno دارسلام DARI SALAAM linamaanisha NYUMBA YA AMANI. Lakini lilipokopwa na kuingizwa kwenye lugha ya Kiswahili, maana yake ilibadilika na ikawa si NYUMBA tena bali sehemu уа јии уа nyumba iliyo chini ya paa.
Alhamisi
ni neno la Kiswahili ambalo lilikopwa kutokana na neno la Kiarabu ASIIMAHKL ألخميس. Kulingana na mpangilio wa siku za Kiarabu ni kuwa Alkhamiis ni siku ya tano ilihali katika lugha ya Kiswahili ni siku ya sita ya wiki. Hebu tulinganishe siku za Kiarabu na zile za Kiswahili:
Kiswahili
Jumamosi (siku ya kwanza ya juma)
Jumapili (siku ya pili ya juma)
Jumatatu (siku ya tatu ya juma)
Jumanne (siku ya nne ya juma)
Jumatano (siku ya tano ya juma)
Alhamisi (siku ya sita ya juma)
Ijumaa (siku ya kukamilisha juma zima)

\section{Kiarabu}
(yaumusabti) siku ya kupumzika
يو (yaumul-ahad) siku ya kwanza ya juma
يومالإثثين (yaumul-ithnaini) siku ya pili ya juma
(yaumuthulathaai) siku ya tatu ya juma
(yaumul-arbiai) siku ya nne ya juma
(alkhamiis) siku ya tano ya juma
يومالجمعة (yaumuljumu'a) siku ya mwisho ya juma

Kulingana na uainishaji na ulinganishaji wa siku za Kiswahili na za Kiarabu tunaona kuwa siku hizo zilipishana, yaani siku ya kwanza ya Kiswahili (Jumamosi) kwa Kiarabu ni siku ya kupumzika. Na siku ya pili ya Kiswahili ikawa siku ya kwanza ya juma ya Kiarabu nk. Kiswahili kiliazima maneno mawili ALHAMISI na IJUMAA kutoka lugha ya Kiarabu. Lakini neno ALHAMISI ambalo lilimaanisha siku ya tano katika lugha ya Kiarabu, lilipofika kwenye lugha ya Kiswahili lilibadili maana na kuwa siku ya sita ya juma.

\section{Sakafu}

ni neno la Kiswahili liliazimwa kutoka lugha ya Kiarabu سقف (saqfu). Neno la Kiarabu saqfu lina maana ya dari, yaani sehemu уа јии уа nуumba iliyo chini ya paa. Lakini neno hili lilipokopwa na kuingizwa ndani ya Kiswahili, maana yake ilibadilika na kuwa sehemu ya chini ya nyumba iliyotandazwa na kutiwa saruji.

\section{Nahau}

nalo ni neno lilioazimwa kutoka lugha ya kiarabu النحو (annahwu) ambalo maana yake asilia katika Kiarabu ni SARUFI. Lakini lilipoingizwa ndani ya Kiswahili, neno hilo lilipata maana nyengine ya fungu la maneno lenye maana maalumu isiyotokana na maana za kawaida za maneno hayo. .

\section{Isimu}

ni neno ambalo pia liliazimwa na kukopwa kutoka lugha ya Kiarabu الإسم (al-ismu) ambalo maana yake asilia ni JINA. Neno hili lilipokopwa na kutiwa Kiswahilini lilibadilishwa maana na kuwa taaluma inayochunguza lugha na kuchambua lugha kisayansi. 


\section{Waraka}

pia nalo llikopwa kutoka lugha ya Kiarabu ورقة (waraqatu). Maana asilia ya neno hili katika lugha ya Kiarabu ni JANI LA MTI. Lakini lilipokopwa na kutiwa kwenye lugha ya Kiswahili, maana yake ilibadilishwa na kuwa hati zilizoandikwa kwenye kipande cha karatasi zenye kutoa habari fulani.

\section{Hitimisho}

makala haya yalitazama na kuchanganua mabadiliko mbalimbali ya kimatamshi ya maneno ya Kiarabu yaliyokopwa na kutiwa kwenye Kiswahili. baada ya kuainisha mabadiliko mbalimbali, iling'amuliwa kuwa maneno mengi ya Kiarabu yaliswahilishwa baada ya kukopwa na konsonanti zake zikabadilishwa ili kurahisisha matamshi kwa wazungumzaji wa Kibantu. mbali na hayo kuna sauti na mpangilio wa matamshi ambao uliazimwa moja kwa moja kutoka lugha ya Kiarabu bila kubadilisha chochote.

Aidha, pia kulitokea mabadiliko ya maana katika baadhi ya maneno ya Kiarabu yaliyokopwa na kutiwa kwenye lugha ya Kiswahili. Kwahivyo, ithibati inaonyesha kuwa kulitokea mabadiliko ya maana pamoja na ya utamkaji wa maabdhi ya maneno ya Kiswahili yaliyokopwa kutoka kwenye lugha ya Kiarabu.

\section{Marejeleo:-}

1. Khamisi A. B. (2012) Kamusi Asisi ya Kiingereza-Kiswahili-Kiarabu; Dar es Salaam; Taasisi ya Taaluma za Kiswahili (TATAKI)

2. Mulder J. \& Hervey S.G. (1975) "Language As a System of Systems" Linguistique, 11, 2, 3-22, 75

3. OUP (2004) Kamusi ya Kiswahili Sanifu (Toleo la Pili); Nairobi; Oxford University Press

4. Paradis, C. (2012).'Lexical Semantics', In TheEncyclopaedia of Applied Linguistics (Ed.),Chappelle C. Oxford: Willey -Blackwell (pp.3347-3356). 